\section{Interventional progress}

\section{S31 SAFETY AND YIELD OF PHYSICIAN LED ULTRASOUND GUIDED TRANSTHORACIC LUNG/PLEURAL BIOPSIES}

R Reddy, M Naeem, G Tsaknis. Kettering General Hospital, Kettering, UK

\subsection{6/thoraxjnl-2015-207770.37}

Introduction Transthoracic ultrasound is important tool in assessing pleural effusions and guiding placement of chest drains. It also demonstrates pleural-based masses and lung tumours abutting the pleura. ${ }^{1}$ Such lesions are suitable for ultrasound guided full core needle biopsy. ${ }^{1}$ Percutaneous transthoracic lung biopsy with ultrasound guidance is not widely performed by respiratory physicians. $^{1}$

Objective To assess safety and yield of ultrasound guided transthoracic biopsy performed by respiratory physicians.

Methods The procedures were carried out in an outpatient or bed side setting between April 2014 and Jun 2015. Apart from checking clotting and omitting antiplatelet/anticoagulants no special prior preparations were undertaken. Under real time transthoracic ultrasound, lesions involving pleura or abutting the pleura which were $>1.5 \mathrm{~cm}$ were sampled 2-3 times with a full core biopsy needle (Biopince 18G). Repeat thoracic ultrasound was done after 10 min to check for pneumothorax. Patients were discharged home around $30 \mathrm{~min}$ post procedure.

Results 51 patients underwent full core biopsy for suspected peripheral lung (44), pleural based (6) and Mediastinal tumours (1). The biopsies were considered adequate in 47 cases (94\%). Diagnosis was achieved in 43 patients with an overall yield of $84 \%$ (Table 1) whilst the yield for malignancy was $82 \%(36 / 44)$. Of the 8 patients with a negative biopsy, malignancy was diagnosed at surgery in 2 patients and CTGB (CT guided biopsy) in 6 patients. Complications were minimal with one patient developing a small pneumothorax (2\%) and another had a small subcutaneous hematoma. After the introduction of the service the waiting list for CT guided biopsies in our hospital has been eliminated.

\begin{tabular}{llll}
\multicolumn{2}{l}{ Abstract S31 Table 1} \\
\hline & Malignant & Benign & Nondiagnostic \\
\hline Total & $36(70 \%)$ & $7(14 \%)$ & $8(16 \%)$ \\
$(n=51)$ & & (Vasculitis 1, TB 1, & (All proven malignant \\
& & Non specific inflammation 3, other 2) & later) \\
\hline
\end{tabular}

Conclusion Ultrasound guided peripheral lung/pleural mass biopsy can be performed by trained respiratory physicians with excellent yield and very low complication rate. Used appropriately it reduces the waiting list for CTGB.

\section{REFERENCE}

1 Diacon AH, Schuurmans MM, Theron J, et al. Safety and yield of ultrasoundassisted transthoracic biopsy performed by pulmonologists. Respiration 2004;71 (5):519-22 S32 VIRTUAL BRONCHOSCOPIC NAVIGATION FOLLOWED BY
RADIAL EBUS TO BIOPSY PERIPHERAL PULMONARY LESIONS: A PILOT STUDY

N Denny, J Mills, L Brown, SJ Fowler, M Munavvar. Department of Respiratory Medicine, Lancashire Teaching Hospitals NHS Foundation Trust, Preston, UK

\subsection{6/thoraxjnl-2015-207770.38}

Introduction Detection of peripheral pulmonary lesions (PPLs) is on the rise but an accurate means of obtaining a tissue diagnosis without high risk of complications is lacking. ${ }^{1}$ Virtual bronchoscopic navigation (VBN) guides the bronchoscope under direct vision and, in combination with radial endobronchial ultrasound (R-EBUS) or ultrathin bronchoscopy, may enhance the diagnostic yield of PPLs with a minimal complication rate. ${ }^{2}$

Aims To pilot the use of VBN in the diagnosis of PPLs. To identify patient and lesion characteristics that predict successful VBN. Methods Images from chest CT (slice width 1-1.25 mm) were acquired from patients, and lesion features, including location and presence of a bronchus sign (bronchus contained within PPL), were recorded. CT images were transferred to a portable workstation and Lungpoint Broncus (C) was used to create a virtual pathway to the PPL. Bronchoscopy was performed with VBN followed by REBUS guidance, under conscious sedation, and biopsies obtained. Pre-procedure characteristics, biopsy adequacy, biopsy outcome, 30-day follow up and complications were recorded.

Results The median age of our cohort $(\mathrm{n}=7)$ was 79 and all patients had one or more comorbidities. PPL median size was 28 $\mathrm{mm}$ and all were located in sub-segmental bronchi. VBN guided the operator to the correct site in six cases. Adequate biopsies were taken from five patients; four had a positive bronchus sign. Three adequate biopsies received a diagnosis of primary lung cancer; those remaining were negative. One patient with inadequate biopsies underwent transthoracic needle biopsy (TTNB) and was diagnosed with primary lung cancer. No complications occurred in spite of the significant co-morbidity of this patient cohort.

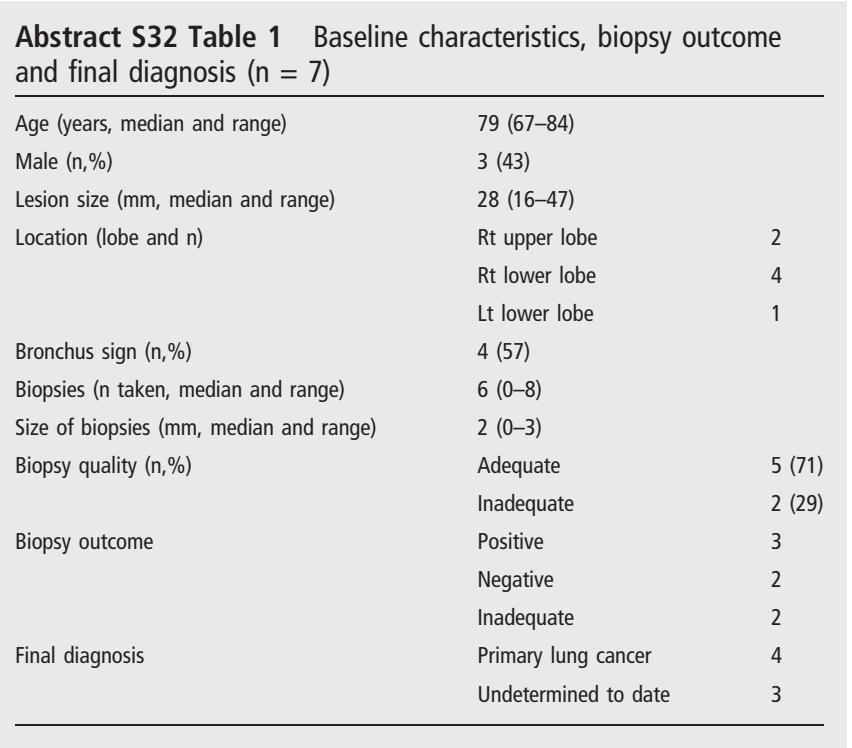

Discussion Our preliminary data suggest that in a selected patient cohort with a bronchus sign, VBN may be a useful and safe adjunct to R-EBUS to obtain a tissue diagnosis from PPLs. In contrast, TTNB has a high diagnostic sensitivity but is 
tarnished by a high complication rate and is not suitable in patients with significant co-morbidity. Therefore, VBN and REBUS are particularly useful where TTNB carries a high risk.

\section{REFERENCES}

1 Kikuchi E, Yamazaki K, Sukoh $N$, et al. Endobronchial ultrasonography with guide-sheath for peripheral pulmonary lesions. Eur Respir J 2004:24:533-7

2 Ishida T, Asano F, Yamazaki K, et al. Virtual bronchoscopic navigation combined with endobronchial ultrasound to diagnose small peripheral pulmonary lesions: a randomised trial. Thorax 2011;66:1072-7

\section{S33 PERFORMANCE OF EBUS-TBNA IN THE PATHOLOGICAL SUBTYPING AND MOLECULAR TESTING OF NON-SMALL CELL LUNG CANCER (NSCLC) IN A UK THORACIC ONCOLOGY CENTRE}

H Al-Najjar, M Evison, J Martin, P Barber, P Crosbie, R Booton. University Hospital of South Manchester, Manchester, UK

\subsection{6/thoraxinl-2015-207770.39}

Introduction The categorisation of NSCLC into squamous and non-squamous subtypes is an important requirement for the optimisation of patient care as this may modify chemotherapy regimens and direct molecular testing. The lung cancer national audit highlights the need to minimise the rate of NSCLC not otherwise specified (NSCLC-NOS). ${ }^{1}$ The aim of our study was to determine whether samples obtained by endobronchial ultrasound guided-transbronchial needle aspiration (EBUS-TBNA) could be used to pathologically subtype NSCLC and provide sufficient material for molecular testing.

Methods A prospectively maintained database of consecutive patients with suspected lung cancer referred to our unit, a UK regional thoracic oncology centre, was analysed. All patients diagnosed with NSCLC by EBUS-TBNA cytology at our centre between Sept 2013 and Sept 2014 were included in the study. Results A total of 89 patients were diagnosed with NSCLC using EBUS-TBNA. The pathological subtypes were: $\mathrm{n}=46$ (51.7\%) squamous cell carcinoma, $n=41(46 \%)$ adenocarcinoma and $n$ $=2(2.2 \%)$ NSCLC-NOS. All samples with a new diagnosis of non-squamous subtype were sent for EGFR mutation analysis, with sufficient material in 97\% $(\mathrm{n}=35 / 36)$ and one activating mutation was identified. ALK analysis was successfully performed in all 5 samples in which this was requested. Additional molecular testing was requested in 9 samples with sufficient material in $89 \%(\mathrm{n}=8 / 9)$.

Conclusions EBUS-TBNA cytology can be used to successfully subtype NSCLC and provide adequate material for molecular testing in the majority of cases. The rate of NSCLC-NOS in our study $(2.2 \%)$ compares favourably with local cancer network $(13.5 \%)$ and national (12.9\%) figures.

\section{REFERENCE}

1 National lung cancer audit report 2014 doi http://www.hscic.gov.uk/catalogue/ PUB16019/clin-audi-supp-prog-lung-nlca-2014-rep.pdf

\section{S34 DO BRONCHIAL WASHINGS IMPROVE THE DIAGNOSTIC SENSITIVITY FOR LUNG CANCER WHEN ENDOBRONCHIAL TUMOUR IS SEEN?}

A Dewar, S Bartlett, R Breen. Guy's and St Thomas NHS Foundation Trust, London, UK

10.1136/thoraxjnl-2015-207770.40
Background/introduction Current BTS guidelines suggest that when endobronchial tumour is seen optimal diagnostic sensitivity is achieved when at least five mucosal biopsies are supplemented with bronchial washings and brushings.

We review our bronchoscopy practice annually in line with current guidance, and strive to make continuous improvements. We have previously noted that bronchial brushings improve the diagnostic sensitivity for lung cancer when an endobronchial tumour is seen, but bronchial washing samples do not.

Aims To confirm that bronchial washings do not increase diagnostic sensitivity for lung cancer where endobronchial tumour is seen at flexible bronchoscopy.

Method We reviewed all flexible bronchoscopy procedures performed at our hospital during a two-year period $(\mathrm{n}=365)$. We reviewed the Electronic Patient records for histology and cytology results in all cases where endobronchial tumour was visualised.

Results Mucosal biopsies and either bronchial brushings or bronchial washings or brushings and washings were performed in all cases where an endobronchial lesion was seen $(n=65)$. Washings were performed in addition to mucosal biopsies in $95 \%$ of cases, bronchial brushings however were sent in $78 \%$ of cases.

The diagnostic sensitivity for mucosal biopsies alone was $80 \%$ $(\mathrm{n}=65)$, bronchial brushings in addition to mucosal biopsies improved diagnostic sensitivity to $86 \%$. In the small number of cases $(n=4)$ mucosal biopsies were negative for malignancy but a malignant diagnosis made on bronchial brushings and washings, bronchial brushings were positive in all cases and whereas bronchial washings were positive in only $50 \%$.

Conclusion Bronchial washings did not add any additional value to the diagnosis of lung cancer when endobronchial tumour was seen. We suggest that mucosal biopsies and brushings combined provide optimal diagnostic sensitivity in these cases. Omitting bronchial washing would produce both cost saving (in our Trust processing bronchial washings costs $£ 76.50$ per sample), and time efficiencies; bronchoscopists could then focus on obtaining multiple good quality mucosal biopsies, which are of paramount importance in molecular subtyping of lung cancers.

\section{S35 METHYLENE BLUE STAINING DIFFERENTIATES NON- SMALL CELL LUNG CANCER TISSUE}

${ }^{1} \mathrm{P}$ Riddell, ${ }^{2} \mathrm{EL}$ Molloy, ${ }^{2} \mathrm{~S}$ Finnegan, ${ }^{1} \mathrm{EP}$ Judge, ${ }^{1} \mathrm{KC}$ Redmond, ${ }^{1} \mathrm{~N}$ Mulligan, ${ }^{2} \mathrm{M}$ Maguire, ${ }^{2} \mathrm{~S}$ O'Dea, ${ }^{1} \mathrm{JJ}$ Egan. ${ }^{1}$ Mater Misericordiae University Hospital, Dublin, Ireland; ${ }^{2}$ Maynooth University, Maynooth, Ireland

\subsection{6/thoraxjnl-2015-207770.4}

Introduction The early detection of lung cancer during bronchoscopy remains a diagnostic challenge. Chromobronchoscopy, using vital dyes, has the potential to aid diagnosis by highlighting areas of dysplastic or malignant change. There are limited numbers of studies in this field but results to date are conflicting. Using a novel electrospray system, we delivered targeted methylene blue (MB) to ex vivo human lung cancer tissue. The aim of this study was to identify whether MB provided a differential stain for lung cancer.

Methods Patients undergoing surgical resection were consented to the study. Following lobectomy, fresh sections of cancerous and non-cancerous tissue were obtained. A range of concentrations of $\mathrm{MB}$ were applied topically to tissue sections by electrospray atomisation. Following delivery of $\mathrm{MB}$, the tissue was washed with $0.9 \%$ saline and images captured. Results were classified in terms of intensity of dye uptake as well as differential staining between normal and cancerous tissue. 